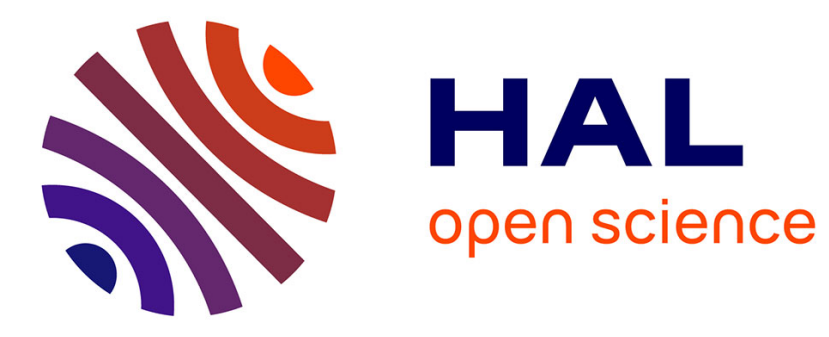

\title{
Power partitions and a generalized eta transformation property
}

Don Zagier

\section{To cite this version:}

Don Zagier. Power partitions and a generalized eta transformation property. Hardy-Ramanujan Journal, 2022, Special commemorative volume in honour of Srinivasa Ramanujan - 2021, Volume 44 - Special Commemorative volume in honour of Srinivasa Ramanujan - 2021, pp.1 - 18. 10.46298/hrj.2022.8932 . hal-03494849

\section{HAL Id: hal-03494849 \\ https://hal.science/hal-03494849}

Submitted on 20 Dec 2021

HAL is a multi-disciplinary open access archive for the deposit and dissemination of scientific research documents, whether they are published or not. The documents may come from teaching and research institutions in France or abroad, or from public or private research centers.
L'archive ouverte pluridisciplinaire HAL, est destinée au dépôt et à la diffusion de documents scientifiques de niveau recherche, publiés ou non, émanant des établissements d'enseignement et de recherche français ou étrangers, des laboratoires publics ou privés. 


\title{
Power partitions and a generalized eta transformation property
}

\author{
Don Zagier
}

Dedicated to the memory of Srinivasa Ramanujan

\begin{abstract}
In their famous paper on partitions, Hardy and Ramanujan also raised the question of the behaviour of the number $p_{s}(n)$ of partitions of a positive integer $n$ into $s$-th powers and gave some preliminary results. We give first an asymptotic formula to all orders, and then an exact formula, describing the behaviour of the corresponding generating function $P_{s}(q)=\prod_{n=1}^{\infty}\left(1-q^{n^{s}}\right)^{-1}$ near any root of unity, generalizing the modular transformation behaviour of the Dedekind eta-function in the case $s=1$. This is then combined with the Hardy-Ramanujan circle method to give a rather precise formula for $p_{s}(n)$ of the same general type of the one that they gave for $s=1$. There are several new features, the most striking being that the contributions coming from various roots of unity behave very erratically rather than decreasing uniformly as in their situation. Thus in their famous calculation of $p(200)$ the contributions from arcs of the circle near roots of unity of order 1, 2,3, 4 and 5 have 13, 5, 2, 1 and 1 digits, respectively, but in the corresponding calculation for $p_{2}(100000)$ these contributions have $60,27,4,33$, and 16 digits, respectively, of wildly varying sizes.
\end{abstract}

Keywords. partitions into powers, Hardy-Ramanujan partition formula, circle method.

2010 Mathematics Subject Classification. 05A18, 11P82.

\section{Introduction}

In their famous paper "Asymptotic formulæ in combinatory analysis" [2], Hardy and Ramanujan generalized the classical Euler partition function by considering the "square partition function" $p_{2}(n)$ defined by the generating function

$$
P_{2}(q):=\prod_{m=1}^{\infty}\left(1-q^{m^{2}}\right)^{-1}=: \sum_{n=0}^{\infty} p_{2}(n) q^{n},
$$

and more generally the " $s$-th power partition function" $p_{s}(n)$ defined by the generating function

$$
P_{s}(q):=\prod_{m=1}^{\infty}\left(1-q^{m^{s}}\right)^{-1}=: \sum_{n=0}^{\infty} p_{s}(n) q^{n},
$$

for any positive integer $s$, where $q$ is a variable in the complex unit disk $|q|<1$. In order to study the asymptotics of $p_{s}(n)$ for large $n$, one needs to understand the asymptotic behaviour of $P_{s}(q)$ for $q$ near to 1 , and also, for more detailed asymptotic information including lower-order oscillatory contributions, for $q$ near any root of unity. Hardy and Ramanujan carried out the first step in their paper and gave in particular the asymptotic formula

$$
\log p_{s}(n) \sim(s+1)\left\{\frac{1}{s} \Gamma\left(1+\frac{1}{s}\right) \zeta\left(1+\frac{1}{s}\right)\right\}^{s /(s+1)} n^{1 /(s+1)}
$$

for the growth of $\log p_{s}(n)$ as $n$ tends to infinity.

The function $P_{1}(q)$ is related to the Dedekind eta-function $\eta(\tau)(\tau \in \mathfrak{H}=$ complex upper halfplane) by $\eta(\tau)=\mathbf{e}(\tau / 24) P_{1}(\mathbf{e}(\tau))^{-1}$, where $\mathbf{e}(z)=e^{2 \pi i z}$ as usual, and Hardy and Ramanujan could 
obtain their famous very precise formula for the partition function (later refined by Rademacher to give an exact formula) by exploiting the modular transformation properties of the latter. For integers $s>1$, however, the generalized eta-function $\eta_{s}(\tau)$ defined by

$$
\eta_{s}(\tau)=\mathbf{e}\left(-\frac{1}{2} \zeta(-s) \tau\right) \prod_{m=1}^{\infty}\left(1-\mathbf{e}\left(m^{s} \tau\right)\right) \quad\left(\tau \in \mathfrak{H}, s \in \mathbb{R}_{>0}\right)
$$

does not transform in any way under the modular group, so that here one can a priori use only the Euler-Maclaurin formula or its variants to obtain approximate information about its behaviour near rational arguments, thus obtaining only much weaker asymptotic formulas like (3). However, it turns out that the functions $\eta_{s}$ also satisfy exact identities for all integral values of $s>1$ that give a complete description of their behaviour near rational arguments (and hence in principle the possibility of finding very precise or even exact formulas for $p_{s}(n)$, although we do not carry this out completely in this paper). For instance, we will show that in analogy with the modular functional equation $\eta(-1 / \tau)=\sqrt{\tau / i} \eta(\tau)$ of the Dedekind eta-function, we have

$$
\eta_{2}(-1 / \tau)=\sqrt{2 \pi \tau / i} \eta_{1 / 2}(\sqrt{\tau}) \eta_{1 / 2}(i \sqrt{\tau})
$$

(notice that the definition (4) makes sense even when the index $s$ is non-integral), and similarly

$$
\eta_{s}(-1 / \tau)=(2 \pi)^{(s-1) / 2} \sqrt{\tau / i} \prod_{\substack{z \in \mathfrak{H} \\ z^{s}= \pm \tau}} \eta_{1 / s}(z)
$$

for all positive integers $s$, now with $s$ terms in the product rather than only two as in (5).

More generally, the transformation behaviour of $\eta(\tau)$ under arbitrary elements of the modular group implies that $\eta(\kappa+i / T) / \sqrt{T}$ for a fixed rational number $\kappa$ and $T$ tending to infinity is expressible as a convergent infinite sum of pure exponentials in $T$, i.e., as a sum of the form $\sum A_{\nu} e^{\lambda_{\nu} T}$ with some exponents $\lambda_{\nu}$ and some coefficients $A_{\nu}$. Our main result generalizes both that statement and equations (5) and (6):

Theorem 1. The function $\eta_{s}\left(\kappa+i / T^{s}\right) / T^{s / 2}$ for every natural number $s$ and every rational number $\kappa$ can be expressed as a convergent infinite sum of pure exponentials in $T$.

The exact form of the expansion asserted by this theorem is much more complicated than the special case $\kappa=0$ as given in (6), and will emerge in conjunction with its proof in the course of the paper. First, however, we will explain in $\S 2$ how one can guess this formula numerically in any particular case, taking $s=2$ and $\kappa$ with denominator 5 as our working example. The next section describes how to obtain the asymptotic expansion of $\eta_{s}\left(\kappa+i / T^{s}\right)$ to all orders in $1 / T$ for any rational number $\kappa$ by splitting the infinite product in (4) into finitely many subproducts according to the value of $m$ modulo the denominator of $\kappa$ and then studying the logarithm of each of these functions by a shifted version of the Euler-Maclaurin formula. This leads to formulas that are not only more precise, but also simpler both to formulate and to prove, than the ones for the full product. We will explain these calculations first in the case $s=1$, even though the result is known there anyway by virtue of the modularity of $\eta(\tau)$, because the method of calculation that is used gives a somewhat different perspective on the behaviour of $\eta(\tau)$ than the usual one and also extends easily to higher values of $s$. The key point for both $s=1$ and the general case is that the logarithms of the individual subproducts have asymptotic expansions that are factorially divergent power series in $1 / T$, but that when we combine them in pairs according to the value of $\pm m$ modulo the denominator of $\kappa$, all but finitely many terms of these expansions cancel and we are left with only terminating series which then are well-defined functions. In $\S 4$ we show how this finite expression can be upgraded to an exact rather than merely asymptotic formula for $\log \eta_{s}\left(\kappa+i / T^{s}\right)$ by the addition of an explicit infinite linear combination of exponentially small terms. Theorem 2 gives this exact formula for the individual 
symmetrized subproducts of $P_{s}(q)$, which are generalizations of the classical Jacobi theta functions as given by their Jacobi triple product expansions, and Theorem 1 in a more precise quantitative version (stated for $s=2$ as Theorem 3) follows easily from this. Finally, in $\S 5$ we study the large $n$ behaviour of the power partition function $p_{s}(n)$ by combining the results of the preceding sections with the Hardy-Ramanujan circle method. The description turns out to be much more complicated than in the original case $s=1$, with the size of the contributions from the neighbourhoods of different rational points ("minor arcs") varying in an unpredictable manner rather than depending only on the denominator of the rational point as in the classical case.

\section{Numerical results}

We originally found equations (5) and (6) by numerical experimentation. Since the right way of doing these experiments is not obvious and will become crucial when we try to generalize (6) to the behaviour of $\eta_{s}$ near arbitrary rational arguments, we say something about this here. For convenience we work with $P_{s}$ rather than $\eta_{s}$ and concentrate on the case $s=2$.

The first step is to observe that the function defined in (1) has the asymptotic expansion

$$
P_{2}\left(e^{-x}\right) \sim \frac{\sqrt{x}}{2 \pi} e^{\Gamma(3 / 2) \zeta(3 / 2) / \sqrt{x}}(1+\varepsilon(x))
$$

with $\varepsilon(x)=\mathrm{O}\left(x^{N}\right)$ for all $N>0$. This is an easy consequence of a version of the Euler-Maclaurin summation formula, as will be explained in more generality in $\S 3$. To understand the number $\varepsilon(x)$, we compute its value numerically to high precision for many small values of $x$. This is easy because the product (1) converges very rapidly. However, $\varepsilon(x)$ not only decays very rapidly, but is also oscillatory, so that its analytic nature is hard to recognize from a table of values or a graph. But even from a table or a graph, by looking at the frequency of the oscillations and at the values of the functions near its local extrema, we can see that it decays roughly exponentially in $1 / \sqrt{x}$ and oscillates with a period that decreases like $x^{3 / 2}$. This suggests:

First simplification: Change variables by setting $x=2 \pi / T^{2}$.

(Here the factor $2 \pi$ is included to simplify later formulas, and the $T^{2}$ would be $T^{s}$ if we were looking at $\eta_{s}$ for $s>2$.) After making this change of variables, we can recognize numerically without difficulty that $\varepsilon\left(2 \pi / T^{2}\right)$ is equal to high precision (meaning to all orders in $1 / T$ as $T \rightarrow \infty$ ) to the function $A_{1}(T):=2 \cos (\sqrt{2} \pi T) e^{-\sqrt{2} \pi T}$. We then subtract $A_{1}(T)$ from $\varepsilon\left(2 \pi / T^{2}\right)$ and find that the difference is equal to $A_{2}(T):=A_{1}(T \sqrt{2})$ to high precision, and subtracting again and repeating we find that the third term is $A_{3}(T):=A_{1}(T \sqrt{3})$. But the fourth term is then not $A_{1}(2 T)$ as one might now expect, but rather $A_{4}(T):=(1+4 \cos (2 \sqrt{2} \pi T)) e^{-2 \sqrt{2} \pi T}$. The next term is again the expected one $A_{5}(T):=A_{1}(T \sqrt{5})$, but now the difference $\varepsilon\left(2 \pi / T^{2}\right)-\sum_{i=1}^{5} A_{i}(T)$ is much larger than $e^{-\sqrt{12} \pi T}$ and it takes considerably more effort to recognize that to high precision it is given by the more complicated function

$$
B_{1}(T)=(2 \cos ((2+\sqrt{2}) \pi T)+2 \cos ((2-\sqrt{2}) \pi T)) e^{-(2+\sqrt{2}) \pi T} .
$$

If we simply continue this way naively, the successive terms get harder and harder to identify, the problem being that each of the functions $A_{1}, \ldots, A_{5}$ and $B_{1}$, and more generally each new remainder term $g(T)$ obtained by subtracting from $\varepsilon\left(2 \pi / T^{2}\right)$ the contributions already identified, turns out to have the form of a finite sum $\sum c_{\nu} e^{-\lambda_{\nu} T}$ with complex exponents $\lambda_{\nu}$ having the same real part, so that $g(T)$ is the product of a pure decaying exponential function $e^{-\Re\left(\lambda_{\nu}\right) T}$ and a bounded but oscillatory trigonometric function. If there were only one term $g(T) \approx c e^{-\lambda T}$, even with a non-real exponent $\lambda$, then it would be easy to find $e^{\lambda}$ to high precision as the value of $g(T+1) / g(T)$ for some large value of $T$, and then to obtain $c$ numerically as $g(T) e^{\lambda T}$ for the same $\lambda$ and $T$. Luckily, there is an easy 
trick $^{1}$ to reduce to this simple situation:

Second simplification: Let $x$ tend to 0 through complex numbers with a small fixed non-zero argument, rather than through positive real values.

In terms of $T$, this says that we let $|T|$ tend to infinity with the argument of $T$ fixed and small. Then each of the terms in the expected sum $\sum c_{\nu} e^{-\lambda_{\nu} T}$ has a different exponential order of growth, so that one of them will dominate all the others, and we can find this one as above and then the further ones inductively by subtracting off one term at a time. In this way it becomes very easy to recognize the precise forms of the next several terms in the expansion of $\varepsilon(T)$, and doing this for the first 15 terms we find that $\varepsilon\left(2 \pi / T^{2}\right)$ is given up to precision $\mathrm{O}\left(e^{-\sqrt{22.3} \pi T}\right)$ by

$$
\varepsilon\left(2 \pi / T^{2}\right)=\sum_{m=1}^{11} A_{m}(T)+\sum_{m=1}^{4} B_{m}(T)+\mathrm{O}\left(e^{-\sqrt{22.3} \pi T}\right)
$$

with

$$
A_{m}(T)=e^{-\sqrt{2 m} \pi T} \times \begin{cases}2 \cos (\sqrt{2 m} \pi T) & \text { for } m \text { squarefree } \\ 4 \cos (\sqrt{2 m} \pi T)+1 & \text { for } m=4 \text { or } m=8 \\ 6 \cos (\sqrt{2 m} \pi T)+4 \cos (\sqrt{2 m} \pi T / 3) & \text { for } m=9\end{cases}
$$

and with $B_{2}, B_{3}$ and $B_{4}$ (we have already given $B_{1}$ ) defined by

$$
\begin{aligned}
& B_{2}(T)=(2 \cos ((\sqrt{6}+\sqrt{2}) \pi T)+2 \cos ((\sqrt{6}-\sqrt{2}) \pi T)) e^{-(\sqrt{6}+\sqrt{2}) \pi T}, \\
& B_{3}(T)=(2 \cos ((\sqrt{6}+2) \pi T)+2 \cos ((\sqrt{6}-2) \pi T)) e^{-(\sqrt{6}+2) \pi T} \\
& B_{4}(T)=(2 \cos ((\sqrt{10}+\sqrt{2}) \pi T)+2 \cos ((\sqrt{10}-\sqrt{2}) \pi T)) e^{-(\sqrt{10}+\sqrt{2}) \pi T} .
\end{aligned}
$$

It is still not easy to recognize the general pattern and to guess the next terms. However, here again the solution is very simple:

Third simplification: Study $\log (1+\varepsilon(x))$ numerically rather than $\varepsilon(x)$ itself, i.e., work with the logarithm of $P_{s}(q)$ or of $\eta_{s}(\tau)$ rather than with these functions themselves.

When we do this in our example, all of the mystery disappears: the whole sum is now just a linear combination of the functions $e^{-\sqrt{2 n} \pi T} \cos (\sqrt{2 n} \pi T)$ with $n \in \mathbb{N}$, with the more complicated exponents involving numbers like $\sqrt{6}+\sqrt{2}$ that we found above arising simply as products of these when we exponentiate. Moreover, by looking at the first few terms, we find that the coefficient of $e^{-\sqrt{2 n} \pi T} \cos (\sqrt{2 n} \pi T)$ is always 2 for $n$ squarefree, is 3 if $n$ is 4 times a squarefree number, and in general is equal to $2 \sigma_{-1}(\sqrt{n})$, where $\sigma_{-1}(\sqrt{n}) \in \mathbb{Q}$ denotes the sum of the reciprocals of the positive integers $d$ with $d^{2} \mid n$. This leads to the guess

$$
\varepsilon\left(2 \pi / T^{2}\right) \approx 2 \sum_{n=1}^{\infty} \sigma_{-1}(\sqrt{n}) e^{-\sqrt{2 n} \pi T} \cos (\sqrt{2 n} \pi T)=-\sum_{m=1}^{\infty} \sum_{ \pm} \log \left(1-e^{-2 \pi i^{ \pm 1 / 2} \sqrt{m} T}\right),
$$

where " $\approx$ " denotes equality up to arbitrarily small exponential terms. But when we compute the (rapidly convergent) right-hand side of this equation, we find that it is not only very nearly, but exactly equal to the numerical value of the left-hand side for every $T$, and exponentiating this formula we indeed find (5), the only slightly subtle point being that to make the coefficients of $\sqrt{T}$ in the exponents match we have to use the functional equation of the Riemann zeta function to write

\footnotetext{
${ }^{1}$ found in connection with a joint paper with Stavros Garoufalidis [1] on quantum knot invariants that has some formal similarities with the results of this note
} 
$(2 \pi)^{-1 / 2} \Gamma(3 / 2) \zeta(3 / 2)$ as $-\pi \sqrt{2} \zeta(-1 / 2)$. Exactly the same type of calculation for $P_{s}$ with $s>2$ then leads eventually to (6), the only difference being that now (7) is replaced by

$$
P_{s}\left(e^{-x}\right) \sim \frac{\sqrt{x}}{(2 \pi)^{s / 2}} e^{-\Gamma(1+1 / s) \zeta(1+1 / s) x^{-1 / s}+\zeta(-s) x / 2}\left(1+\varepsilon_{s}(x)\right)
$$

with $\varepsilon_{s}(x)$ of rapid decay as $x \rightarrow 0$ and we have to use the functional equation of the Riemann zeta function to write $(2 \pi)^{-(s-1) / 2} \Gamma(1+1 / s) \zeta(1+1 / s)$ as an algebraic multiple of $2 \pi \zeta(-1 / s)$.

This completes our numerical discussion of the functional equations (5) and (6). We can now use the experience we have gained to look also at the values of $P_{s}(q)$ near roots of unity. As an example we consider $P_{2}$ near fifth roots of unity. Here a more complicated version of the Euler-Maclaurin formula, explained in detail in $\S 3$, leads to the asymptotic formula

$$
P_{2}\left(\zeta_{5}^{a} e^{-x}\right) \sim \frac{5}{2 \pi} \sqrt{x} e^{C_{a / 5} / \sqrt{x}}\left(1+\varepsilon_{2, a / 5}(x)\right) \quad\left(a \in(\mathbb{Z} / 5 \mathbb{Z})^{\times}\right)
$$

with $\varepsilon_{2, a / 5}(x)$ of rapid decay (i.e., $\mathrm{O}\left(x^{N}\right)$ for all $N>0$ ) as $x$ tends to zero, where $\zeta_{5}=\mathbf{e}\left(\frac{1}{5}\right)$ and $C_{a / 5}=\frac{\sqrt{\pi}}{2 \sqrt{5}}\left(\frac{1}{5} \zeta\left(\frac{3}{2}\right)+\left(\frac{a}{5}\right) L\left(\frac{3}{2},\left(\frac{\dot{5}}{5}\right)\right)\right)$. (Here $L\left(s,\left(\frac{\dot{5}}{5}\right)\right.$ is the Dirichlet $L$-series associated to the Legendre symbol $(\dot{\overline{5}})$.) Finding an exact formula for $\varepsilon_{2, a / 5}(x)$ by a direct additive approach would be essentially hopeless, but using the same three tricks as above (setting $x=2 \pi / T^{2}$, approaching zero at an angle, and taking the logarithm), we easily discover that the logarithm of $1+\varepsilon_{a, 2 / 5}(x)$ is given as a linear combination of the pure exponential functions $e^{-2 \pi^{3 / 2}(1 \pm i) \sqrt{m / 125 x}}$ with $m \in \mathbb{N}$, and we can compute the successive coefficients numerically without too much difficulty. However, when we do this they turn out to be quite hard to recognize, since the nature of the coefficient turns out to be different for $m$ prime to $5, m$ exactly divisible by 5 , and $m$ divisible by 25 . Only after computing 300 coefficients can we guess the law of formation with some confidence:

$$
-\log \left(1+\varepsilon_{2, a / 5}(x)\right)=G_{a}\left(\frac{2 \pi^{3 / 2}(1+i)}{\sqrt{125 x}}\right)+G_{-a}\left(\frac{2 \pi^{3 / 2}(1-i)}{\sqrt{125 x}}\right) \quad(x \rightarrow 0),
$$

where

$$
G_{a}(X)=\sum_{m=1}^{\infty} \sigma_{-1}(\sqrt{m}) D\left(a^{-1} m\right) e^{-\sqrt{m} X}+\frac{1}{5} \sum_{m=1}^{\infty}\left(\frac{a^{-1} m}{5}\right) \log \left(1-e^{-5 \sqrt{m} X}\right)
$$

with $\sigma_{-1}(\sqrt{m})$ as above and $D: \mathbb{Z} / 5 \mathbb{Z} \rightarrow \mathbb{R}$ defined by $D(0)=-1, D( \pm 1)=\frac{1 \pm \sqrt{5}}{2}, D( \pm 2)=0$.

As a final comment, we note that the constant $C_{a, 5}$ in the exponent in equation (9) is positive for $a \equiv \pm 1(\bmod 5)$ but negative for $a \equiv \pm 2(\bmod 5)$, so that the function $\eta_{2}$, unlike $\eta_{1}=\eta$, is not always exponentially small near rational values of its argument.

\section{Asymptotics near rational points}

In order to make sense of the coefficients of the expansion of $\eta_{s}(\tau)$ near a rational point, it turns out to be useful to refine the statement by breaking up the infinite product defining $\eta_{s}(\tau)$ into finitely many subproducts and studying the asymptotics of each one separately, since the formulas for these are easier to recognize, easier to prove, and also stronger than the ones for the whole product. Above all, this approach combined with a combination of the subproducts into pairs will produce terminating asymptotic expressions and later (in $§ 4$ ) will lead to exact rather than merely asymptotic expressions for $\eta_{s}(\tau)$.

We start by explaining this in detail in the case $s=1$, where everything is known due to the modularity properties of the Dedekind eta-function. Here it is more convenient to work with $\eta=\eta_{1}$ 
rather than with $P_{1}$, since the action of the modular group can be seen more clearly. The fact that $\eta(\tau)^{24}$ is a modular form of weight 12 on $\mathrm{SL}(2, \mathbb{Z})$ implies that

$$
\eta\left(\frac{a \tau+b}{c \tau+d}\right)=\mathbf{e}\left(\frac{N(\gamma)}{24}\right)(c \tau+d)^{1 / 2} \eta(\tau) \quad \forall \gamma=\left(\begin{array}{ll}
a & b \\
c & d
\end{array}\right) \in \operatorname{SL}(2, \mathbb{Z})
$$

where $(c \tau+d)^{1 / 2}$ denotes the principal branch and $N(\gamma)$ is an integer depending on $\gamma$. Of course one can prove this by checking it only when $\gamma$ is one of the standard generators $\left(\begin{array}{ll}1 & 1 \\ 0 & 1\end{array}\right)$ (for which it is trivial) and $\left(\begin{array}{cc}0 & -1 \\ 1 & 0\end{array}\right)$ (for which several ad hoc proofs are known) of $\operatorname{SL}(2, \mathbb{Z})$, but since there is no reasonable action of $\mathrm{SL}(2, \mathbb{Z})$ on $\eta_{s}(\tau)$ for $s>1$ we want to sketch an argument that does not use the group structure. In fact, this argument proves a stronger statement than (10), namely the transformation law

$$
H\left(\frac{a \tau+b}{c \tau+d}\right)=\frac{N(\gamma)}{24}+\log (c \tau+d)+H(\tau)
$$

where Log denotes the principal branch of the logarithm and

$$
H(\tau)=\frac{\pi i \tau}{12}+\sum_{m=1}^{\infty} \log \left(1-q^{m}\right) \quad(q=\mathbf{e}(\tau))
$$

is the branch of $\log \eta(\tau)$ that is real when $\tau$ is pure imaginary. Of course the formula (11) is well known, going back to Riemann and to Dedekind's paper on some "fragments" of Riemann in which he defined and gave the transformation properties of the function now named after him. But our approach is somewhat different than the standard one and in some ways more elementary, since it does not use the group structure of the modular group, and also demonstrates that the widely-held belief that it would be impossible to even guess, let alone prove, the full expansion of the partition function coming from the circle method without using the modularity of the eta-function, is false.

The first step is to rewrite (10) with arguments $\tau=\frac{-d+i / x}{c}$ and $\frac{a \tau+b}{c \tau+d}=\frac{a+i x}{c}$, so that the behaviour of $\eta$ near $a / c$ corresponds to $\tau$ tending to infinity or $x$ tending to 0 and is more clearly visible. With this substitution the variable $q$ in (12), with $\tau$ replaced by $\frac{a \tau+b}{c \tau+d}$, is equal to $\mathbf{e}(a / c) e^{-2 \pi m x / c}$ and hence very near a $c$ th root of unity, so we have to break up the sum on the right in according to the value of $m(\bmod c)$. This gives

$$
H\left(\frac{a+i x}{c}\right)=\pi i \frac{a+i x}{12 c}-\sum_{\ell(\bmod c)} L\left(\frac{\ell}{c}, \frac{a \ell}{c} ; 2 \pi x\right),
$$

where $L(\alpha, \beta ; t)$ is defined for $\alpha, \beta \in \mathbb{R} / \mathbb{Z}$ and $t$ positive (or complex with positive real part) by

$$
L(\alpha, \beta ; t)=-\sum_{\substack{\nu \in \mathbb{Z}+\alpha \\ \nu>0}} \log \left(1-\mathbf{e}(\beta) e^{-\nu t}\right) .
$$

(The minus sign here is included for convenience, to simplify signs elsewhere.)

If we want only the asymptotics of $L(\alpha, \beta ; t)$ to all orders in $t$ as $t \rightarrow 0$, we can simply use the shifted Euler-Maclaurin formula. This formula, which is discussed in detail in [4], says that, for any smooth function $f$ on $[0, \infty)$ that is small at infinity and for any positive real number $\alpha$ (or complex number $\alpha$ which is not a non-positive real number), the sum $\sum_{n=0}^{\infty} f((n+\alpha) t)$ has a Laurent expansion at $t=0$ (valid to all orders in $t$, though in general not convergent) given by

$$
\sum_{n=0}^{\infty} f((n+\alpha) t) \sim I_{f} t^{-1}+\sum_{r=0}^{\infty} \zeta(-r, \alpha) a_{r} t^{r} \quad \text { as } t \searrow 0,
$$


where $I_{f}=\int_{0}^{\infty} f(x) d x$ (so that the sum on the left is a Riemann sum for the integral $I_{f} / t$ ), the $a_{r}$ are the Taylor coefficients $f^{(r)}(0) / r$ ! of $f(t)$ at $t=0$, and $\zeta(-r, \alpha)$ is the Hurwitz zeta function, defined for $s \in \mathbb{C} \backslash\{1\}$ by meromorphic continuation from its value $\sum_{n=0}^{\infty}(n+\alpha)^{-s}$ for $\Re(s)>1$. The value of $\zeta(s, \alpha)$ at $s=-r$ is equal to $-B_{r+1}(\alpha) /(r+1)$, where $B_{n}(\alpha)$ denotes the $n$th Bernoulli polynomial. We will use formula (15) in its equivalent periodic form

$$
\sum_{\substack{\nu \in \mathbb{Z}+\alpha \\ \nu>0}} f(\nu t) \sim I_{f} t^{-1}+\sum_{r=0}^{\infty} \bar{\zeta}(-r, \alpha) a_{r} t^{r}-\frac{a_{0}}{2} \delta_{\alpha, 0} \quad(t \searrow 0),
$$

where $\alpha$ is now in $\mathbb{R} / \mathbb{Z}$ and where $\bar{\zeta}(s, \alpha)$ is the meromorphic continuation of $\sum_{\nu \in(\mathbb{Z}+\alpha)_{>0}} \nu^{-s}$, whose value at $s=-r$ equals $-\bar{B}_{r+1}(\alpha) /(r+1)$. Here $\bar{B}_{n}(x)$ is the piecewise analytic periodic function on $\mathbb{R}$ defined by $\bar{B}_{0}(x)=1$ and by

$$
\bar{B}_{n}(x)=\frac{(n-1) !}{(2 \pi i)^{n}} \sum_{m \neq 0} \frac{e^{2 \pi i m x}}{m^{n}}
$$

for $n \geq 1$ (for $n=1$ the sum is only conditionally convergent and must be interpreted as a Cauchy principal value), which is equal to $B_{n}(x-[x])$ except when $n=1$ and $x \in \mathbb{Z}$, in which case $\bar{B}_{1}(x)=0$ is the average of $B_{1}(0)=-\frac{1}{2}$ and $B_{1}(1)=\frac{1}{2}$. If we apply this to the function $f(t)=-\log \left(1-\mathbf{e}(\beta) e^{-t}\right)$, for which $I_{f}=\operatorname{Li}_{2}(\mathbf{e}(\beta))$ and $a_{r}=\frac{(-1)^{r}}{r !} \operatorname{Li}_{1-r}(\mathbf{e}(\beta))$ if $\beta \neq 0$, where the polylogarithm $\operatorname{Li}_{k}(z)$ is defined on $\mathbb{C} \backslash[1, \infty)$ by analytic continuation of $\sum_{n=1}^{\infty} z^{n} / n^{k}$ and is a polynomial in $z /(1-z)$ with integral coefficients when $k \leq 0$, we obtain the expansion

$$
L(\alpha, \beta ; t) \sim \frac{\operatorname{Li}_{2}(\mathbf{e}(\beta))}{t}+\sum_{r=0}^{\infty} \frac{\bar{B}_{r+1}(-\alpha)}{(r+1) !} \operatorname{Li}_{1-r}(\mathbf{e}(\beta)) t^{r}
$$

if $\alpha$ and $\beta$ are both non-zero, and a slight modification of this formula if one of them vanishes. In particular, if $\beta=0$ then we must use the modification of the shifted Euler-Maclaurin formula (15), also given in [4], that applies when the function $f(t)$ has a logarithmic singularity at the origin.

Now comes the key point. The power series in $x$ occurring in (18) is factorially divergent, since both the Bernoulli polynomials and the negative-index polylogarithms grow factorially in their index. But if we symmetrize, combining the two values $(\alpha, \beta)$ and $(-\alpha,-\beta)$ in $(18)$ (which is all that is needed for the application to $\log \eta$ since it corresponds to combining the terms $\ell$ and $-\ell$ in (13)), then because of the symmetry properties

$$
\bar{B}_{k}(-\alpha)=(-1)^{k} \bar{B}_{k}(\alpha), \quad \mathrm{Li}_{-k}(1 / x)=(-1)^{k-1} \mathrm{Li}_{-k}(x) \quad(k>0)
$$

of Bernoulli polynomials and negative-index polylogarithms there is a huge simplification and the symmetrized function

$$
L^{\mathrm{sym}}(\alpha, \beta ; t):=L(\alpha, \beta ; t)+L(-\alpha,-\beta ; t)
$$

is given by a terminating Laurent series. Explicitly, we have

$$
L^{\mathrm{sym}}(\alpha, \beta ; t) \sim 2 \pi^{2} \bar{B}_{2}(\beta) t^{-1}+2 \pi i \bar{B}_{1}(\alpha) \bar{B}_{1}(\beta)-\frac{1}{2} \bar{B}_{2}(\alpha) t .
$$

if $\alpha$ and $\beta$ are both different from 0 , and a slight modification of this if one or both of them vanish. Inserting this expansion into (13), we obtain a proof of the formula (11) asymptotically up to all orders in $1 / \tau$ as $\tau \rightarrow \infty$, including an explicit formula for $N(\gamma)$ as a Dedekind sum.

This finishes our discussion of the situation for the original Dedekind eta-function. It is now easy to repeat the whole calculation for $\eta_{s}(t)$ with $s>1$, with $H(t)$ replaced by the function

$$
H_{s}(\tau)=-\pi i \zeta(-s) \tau+\sum_{m=1}^{\infty} \log \left(1-q^{m^{s}}\right)
$$


which again is the branch of the logarithm of $\eta_{s}(\tau)$ that is real on the positive imaginary axis. Arguing exactly as before, we obtain the decomposition

$$
H_{s}\left(\frac{a+i x}{c}\right)=-\pi i \zeta(-s) \frac{a+i x}{c}-\sum_{\ell(\bmod c)} L_{s}\left(\frac{\ell}{c}, \frac{a \ell^{s}}{c} ;\left(2 \pi c^{s-1} x\right)^{1 / s}\right) \quad(x \searrow 0)
$$

of $H_{s}(\tau)$ as $\tau$ approaches a rational point $a / c$, with $L_{s}(\alpha, \beta ; t)$ defined by

$$
L_{s}(\alpha, \beta ; t)=-\sum_{\substack{\nu \in \mathbb{Z}+\alpha \\ \nu>0}} \log \left(1-\mathbf{e}(\beta) e^{-(\nu t)^{s}}\right) .
$$

The asymptotic expansion of $L_{s}(\alpha, \beta ; t)$ as $t$ tends to 0 can be obtained using the same shifted Euler-Maclaurin formula (16) as before, this time applied to $f(t)=-\log \left(1-\mathbf{e}(\beta) e^{-t^{s}}\right)$, for which $I_{f}=\Gamma(1+1 / s) \operatorname{Li}_{1+1 / s}(\mathbf{e}(\beta))$ by an easy calculation (expand $f(t)$ as $\sum_{k>0} k^{-1} \mathbf{e}(k \beta) e^{-k(\nu t)^{s}}$ and integrate termwise) and $a_{r}$ (for $\beta \neq 0$ ) equals $\frac{(-1)^{\ell}}{\ell !} \operatorname{Li}_{1-\ell}(\mathbf{e}(\beta))$ if $r=s \ell$ with $\ell \geq 0$ and vanishes if $s \nmid r$. This leads to an infinite asymptotic formula for $L_{s}(\alpha, \beta ; t)$ generalizing (18) which we do not write out here. Once again the key point is that if we symmetrize $L_{s}$, this time by setting

$$
L_{s}^{\mathrm{sym}}(\alpha, \beta ; t):=L_{s}(\alpha, \beta ; t)+L_{s}\left(-\alpha,(-1)^{s} \beta ; t\right)
$$

then by virtue of the same symmetry relations (19) as before the divergent infinite series of the two separate terms cancel and we are left with a finite expression. We omit the details of the calculation, which are straightforward but fairly messy, but do write out the final formula completely because it involves several case distinctions depending on the parity of $s$ (we already saw this in formula (8), which had an extra term $\zeta(-s) x / 2$ in the exponent that was not visible in (7)) and on whether one or both of $\alpha$ or $\beta$ vanishes.

Proposition 1. Let $s$ be an integer greater than 1 , and $\alpha$ and $\beta$ elements of $\mathbb{R} / \mathbb{Z}$. Then the function $L_{s}^{\text {sym }}(\alpha, \beta ; t)$ defined by equation (24) is given asymptotically by the formula

$$
\begin{aligned}
L_{s}^{\text {sym }}(\alpha, \beta ; t) \sim & \frac{2 \Gamma(1+1 / s)}{t} \times\left\{\begin{aligned}
\operatorname{Li}_{1+1 / s}(\mathbf{e}(\beta)) & \text { if } 2 \mid s \\
\Re\left(\operatorname{Li}_{1+1 / s}(\mathbf{e}(\beta))\right) & \text { if } 2 \nmid s
\end{aligned}\right. \\
+ & \begin{array}{ll}
0 & \text { if } 2 \mid s \\
2 \pi i \bar{B}_{1}(\alpha) \bar{B}_{1}(\beta)+\bar{\zeta}(-s, \alpha) t^{s} & \text { if } 2 \nmid s
\end{array} \\
+ & \begin{array}{ll}
0 & \text { if } \alpha \neq 0, \beta \neq 0 \\
\log (1-\mathbf{e}(\beta)) & \text { if } 2 \mid s, \alpha=0, \beta \neq 0 \\
\log |1-\mathbf{e}(\beta)| & \text { if } 2 \nmid s, \alpha=0, \beta \neq 0 \\
-s \log |1-\mathbf{e}(\alpha)| & \text { if } \alpha \neq 0, \beta=0 \\
s \log (t / 2 \pi) & \text { if } \alpha=\beta=0
\end{array}
\end{aligned}
$$

to all orders in $t$ as $t$ tends to 0.

This proposition has the following consequence for the asymptotics of $P_{s}(q)$ near roots of unity.

Proposition 2. For $s>1$ and $\kappa$ a rational number with denominator $c$ we have

$$
P_{s}\left(\mathbf{e}(\kappa) e^{-1 / T^{s}}\right)=\mathbf{e}\left(D_{s}(\kappa)\right)\left(\frac{N_{s}(c)}{2 \pi T}\right)^{s / 2} e^{C_{s}(\kappa) T+\frac{1}{2} \zeta(-s) T^{-s}} F_{s, \kappa}(T)
$$


with $F_{s, \kappa}(T)=1+O\left(T^{-N}\right)$ for all $N$ as $T$ tends to infinity, where the constant $C_{s}(\kappa)$ is given by

$$
C_{s}(\kappa)=\frac{\Gamma(1+1 / s)}{c} \sum_{\ell(\bmod c)} \operatorname{Li}_{1+1 / s}\left(\mathbf{e}\left(\ell^{s} \kappa\right)\right)
$$

$D_{s}(\kappa)$ is the generalized Dedekind sum $\frac{1}{2} \sum_{\ell(\bmod c)} \bar{B}_{1}(\ell / c) \bar{B}_{1}\left(\ell^{s} \kappa\right)$, and $N_{s}(c)$ is the smallest positive integer whose $s$-th power is divisible by $c$.

The reader can check that the statement of Proposition 2 agrees with equation (9) in the special case $\kappa=a / 5$ and $s=2$ described in connection with the numerical experiments described in $\S 2$, with $C_{\kappa}=C_{2}(\kappa)$ and $N_{2}(5)=5$. However, the generalized Dedekind sum is not visible in that case, since $D_{s}(\kappa)$ vanishes whenever $s$ is even.

\section{Exact formulas}

We now come to the last step, which is the passage from asymptotic to exact formulas for $\eta_{s}(\tau)$ and more generally for $L_{s}^{\mathrm{sym}}(\alpha, \beta ; t)$. Such exact formulas are clear in the case $s=1$ from the modular properties of the Dedekind eta function and more generally of the Jacobi theta function, since the function $L_{1}^{\mathrm{sym}}(\alpha, \beta ; t)$ is essentially equal to the logarithm of the latter by virtue of the famous Jacobi triple product formula. But it is quite surprising that they are still present when these modularity properties no longer hold. In particular, the classical proof of the modularity properties of the Jacobi theta functions is not based on their product expansions, but on their additive representations as theta series (the equality of the two, as found by Jacobi, being an easily proved combinatorial identity), which are lattice sums of pure Gaussians and whose modularity is therefore an easy consequence of the Poisson summation formula and the fact that Gaussian functions are equal to their own Fourier transforms. But for $s>1$ the holomorphic function defined by exponentiating $-L_{s}^{\text {sym }}(\alpha, \beta ; t)$ does not have any reasonable sort of additive expansion, because, as we already saw in $\S 2$ with our numerical experiments and will prove in this section in general, the function $L_{s}^{\mathrm{sym}}(\alpha, \beta ; t)$ for $\beta$ rational is an infinite sum of terms $\widetilde{q} \sqrt[s]{n}$ with $n$ integral and $\widetilde{q}$ fixed, and when $s$ is larger than 1 the exponential of such a sum is no longer a sum of the same type but rather a horrendously complicated linear combination of powers of $\widetilde{q}$ with exponents belong to infinitely many different number fields. The multiplicative argument that we are about to give is therefore of necessity completely different from the standard additive proof of modularity for the case $s=1$ and may be of some interest even in that case.

Before launching into the calculations we make one other observation about the nature of the formulas that we want to obtain. At first sight it might seem unreasonable to expect to be able to go from an asymptotic formula to an exact one, especially since in our case the asymptotic formula, although itself finite, arose by combining two divergent series which do not even have well-defined numerical values. But in fact it turns out that the combined exact formula is actually easier to get than the individual asymptotic ones. The reason is very simple. In the standard Euler-Maclaurin formula, which describes the asymptotics as $x$ tends to 0 of $\sum_{n=1}^{\infty} f(n x)$ for sufficiently nice functions $f$, one is summing over a half-lattice $\mathbb{Z}_{>0}$, and similarly in the shifted Euler-Maclaurin formula one is summing over a half-lattice $(\mathbb{Z}+\alpha)_{>0}$. Sums of this sort are intrinsically difficult because of the jump of the summand at the boundary. But when it is possible to combine two sums over half-lattices into a single sum over a full lattice, shifted or not, then (at least if one is lucky) the summation can be carried out exactly by using the Poisson summation formula, which is more precise and also considerably simpler than the Euler-Maclaurin formula.

In our case this works exactly when $s$ is even, while for odd $s$ a little manipulation will be required. When $s$ is even, we can apply the Poisson summation formula directly because we have

$$
L_{s}^{\mathrm{sym}}(\alpha, \beta ; t)-\delta_{\alpha, 0} \log (1-\mathbf{e}(\beta))=-\sum_{\nu \in \mathbb{Z}+\alpha} \log \left(1-\mathbf{e}(\beta) e^{-(\nu t)^{s}}\right)
$$


where we no longer have to distinguish the cases $\nu>0, \nu<0$ or (if $\alpha=0$ ) $\nu=0$, and where the doubly infinite sum is rapidly convergent. The right-hand side has the form $\sum_{\nu \in \mathbb{Z}+\alpha} f(\nu t)$, where $f(t)=f_{s, \beta}(t)=-\log \left(1-\mathbf{e}(\beta) e^{-t^{s}}\right)$ as before, and hence is equal to $t^{-1} \sum_{m \in \mathbb{Z}} \widetilde{f}\left(m t^{-1}\right) \mathbf{e}(-m \alpha)$ by the Poisson summation formula, where $\widetilde{f}$ denotes the Fourier transform of $f$. The constant term $\widetilde{f}(0)$ is equal to twice the integral $I_{f}$ whose value $\Gamma(1+1 / s) \mathrm{Li}_{1+1 / s}(\mathbf{e}(\beta))$ we already gave, and the terms $m$ and $-m$ for $m>0$ can be combined since $f$ is even. This gives the decomposition

$$
L_{s}^{\mathrm{sym}}(\alpha, \beta ; t)=E_{s}(\alpha, \beta ; t)+\varepsilon_{s}(\alpha, \beta ; t),
$$

where the "elementary term" $E_{s}(\alpha, \beta ; t)$ is given by

$$
E_{s}(\alpha, \beta ; t)=\frac{2 \Gamma(1+1 / s) \operatorname{Li}_{1+1 / s}(\mathbf{e}(\beta))}{t}+\delta_{\alpha, 0} \log (1-\mathbf{e}(\beta))
$$

and coincides with the right-hand side of the asymptotic equation (23) (for simplicity we are assuming here that $\beta \neq 0)$, while the "error term" $\varepsilon_{s}(\alpha, \beta ; t)$ is given by

$$
\varepsilon_{s}(\alpha, \beta ; t)=\frac{1}{t} \sum_{m=1}^{\infty} \tilde{f}\left(\frac{m}{t}\right)(\mathbf{e}(m \alpha)+\mathbf{e}(-m \alpha)) .
$$

In order to compute the Fourier transform $\widetilde{f}(y)$ we integrate once by parts, getting

$$
\widetilde{f}(y)=-\int_{-\infty}^{\infty} \log \left(1-\mathbf{e}(\beta) e^{-t^{s}}\right) \mathbf{e}(t y) d t=\frac{1}{2 \pi i y} \int_{-\infty}^{\infty} \frac{s t^{s-1} \mathbf{e}(t y)}{\mathbf{e}(-\beta) e^{t^{s}}-1} d t .
$$

For $y$ positive, we can move the path of integration upwards, picking up contributions as the residues of the poles of the integrand in the upper half-plane, all of which are simple. The residue of the integrand at $t=x$ with $x^{s} \in 2 \pi i(\mathbb{Z}+\beta)$ is simply $\mathbf{e}(x y) / y$, so we find

$$
\frac{1}{t} \widetilde{f}\left(\frac{m}{t}\right)=\frac{1}{m} \sum_{\substack{x \in \mathfrak{H} \\ x^{s} \in 2 \pi i(\mathbb{Z}+\beta)}} \mathbf{e}\left(\frac{m x}{t}\right) \quad(m>0) .
$$

Inserting this into the formula for $\varepsilon_{s}$ and summing over $m \in \mathbb{N}$, we obtain

$$
\varepsilon_{s}(\alpha, \beta ; t)=-\sum_{\substack{x \in \mathfrak{H} \\ x^{s} \in 2 \pi i(\mathbb{Z}+\beta)}}(\log (1-\mathbf{e}(\alpha+x / t))+\log (1-\mathbf{e}(-\alpha+x / t))) .
$$

We now have to interpret this result. The values of $x^{s}$ in (28) lie on two half-rays, the positive and negative imaginary axes (the value 0 does not occur because we are assuming that $\beta \neq 0$ ), so the values of $x$ lie on $s$ half-rays, namely the $s$-th roots of the positive and negative imaginary axes that lie in the upper half-plane. The contribution of each such half-ray is a sum of terms $-\log \left(1-\mathbf{e}( \pm \alpha) e^{\sqrt[s]{\nu} u}\right)$ with $u$ fixed and $\nu$ ranging over the positive elements of the shifted lattice $\mathbb{Z}+\beta$ or $\mathbb{Z}-\beta$, so (28) expresses $\varepsilon_{s}(\alpha, \beta ; t)$ as a finite linear combination of values of the function $L_{1 / s}$ with suitable arguments. If we write out the identity in this way the result is quite ugly, but it becomes more elegant after a change of variables. The point is that the function $L_{s}(\alpha, \beta ; t)$ as defined in (23) is actually a holomorphic function of $t^{s}$, not just of $t$ itself, and we chose to use $t$ rather than $t^{s}$ as its argument only in order to make the application of the Euler-Maclaurin formula simpler to follow. We now obtain nicer expressions by changing the variable from $t$ to $\tau=i t^{s} / 2 \pi$, and also, for aesthetic reasons, by changing the sign of $L_{s}$ and exponentiating. We therefore define

$$
\Lambda_{s}^{0}(\alpha, \beta ; \tau):=e^{-L_{s}(\alpha, \beta ; \sqrt[s]{2 \pi \tau / i})}=\prod_{\substack{\nu \in \mathbb{Z}+\alpha \\ \nu>0}}\left(1-\mathbf{e}(\beta) q^{\nu^{s}}\right) \quad(\tau \in \mathfrak{H}),
$$


which for fixed $\alpha$ and $\beta$ in $\mathbb{R} / \mathbb{Z}$ is a holomorphic function in the upper half-plane. (The superscript " 0 " here indicates that this is not yet our final normalization, which will be given below.) When $s=1$ the expression $q^{\nu^{s}}$ with $\nu=n+\alpha$ factorizes as $q^{n} \mathbf{e}(z)$ with an elliptic variable $z=\alpha \tau+\beta$, so that $\Lambda_{s}^{0}$ in this case is just the classical $q$-Pochhammer symbol or shifted $q$-factorial $(\mathbf{e}(z) ; q)_{\infty}=\prod_{n=0}^{\infty}\left(1-q^{n} \mathbf{e}(z)\right)$, but for $s>1$ there is no such splitting. The multiplicatively symmetrized version of $\Lambda_{s}^{0}(\alpha, \beta ; \tau)$ obtained by exponentiating $-L_{s}^{\text {sym }}(\alpha, \beta ; t)$, and also multiplying by the constant factor $1-\mathbf{e}(\beta)$ when $\alpha=0$, is then the function

$$
\Theta_{s}(\alpha, \beta ; \tau):=(1-\mathbf{e}(\beta))^{\delta_{\alpha, 0}} \Lambda_{s}^{0}(\alpha, \beta ; \tau) \Lambda_{s}^{0}(-\alpha, \beta ; \tau)=\prod_{\nu \in \mathbb{Z}+\alpha}\left(1-\mathbf{e}(\beta) q^{\nu^{s}}\right),
$$

where the product is now over a complete (shifted) lattice and is absolutely convergent. (This is simply the exponentiated form of equation (26).) We have denoted this function by $\Theta_{s}$ because it can be seen as the analogue for even $s$ of the Jacobi triple product formula for $s=1$. Formula (28), together with (27), now takes on the relatively simple form

$$
\Theta_{s}(\alpha, \beta ; \tau)=e^{c / \tau^{1 / s}} \cdot \prod_{\substack{\mathfrak{z} \in \mathfrak{H} \\ \mathfrak{z}^{s}=-1 / \tau \\ \varepsilon= \pm 1}} \Lambda_{1 / s}^{0}(\beta, \varepsilon \alpha ; \mathfrak{z}) \cdot \prod_{\substack{\mathfrak{z} \in \mathfrak{H} \\ \mathfrak{z}^{s}=1 / \tau \\ \varepsilon= \pm 1}} \Lambda_{1 / s}^{0}(-\beta, \varepsilon \alpha ; \mathfrak{z})
$$

for an appropriate explicit constant $c$.

If $s$ is odd, then the calculation above fails because replacing the sum over $\nu>0$ in (23) by a sum over all $\nu \in \mathbb{Z}+\alpha$ would now give a divergent expression. The value of $L_{s}^{\text {sym }}(\alpha, \beta ; t)$, modified as before by adding a term $-\log (1-\mathbf{e}(\beta))$ if $\alpha=0$, is given instead by

$$
L_{s}^{\mathrm{sym}}(\alpha, \beta ; t)-\delta_{a, 0} \log (1-\mathbf{e}(\beta))=-\sum_{\nu \in \mathbb{Z}+\alpha} \begin{cases}\log \left(1-\mathbf{e}(\beta) e^{-\nu^{s} t^{s}}\right) & \text { if } \nu \geq 0, \\ \log \left(1-\mathbf{e}(-\beta) e^{\nu^{s} t^{s}}\right) & \text { if } \nu<0,\end{cases}
$$

which is once again a sum over the whole (shifted) lattice but to which Poisson summation cannot be applied directly because the summands for $\nu \geq 0$ and $\nu<0$ are different. However, they differ only by the addition of a linear combination of 1 and $t^{s}$, or of 1 and $\tau$ after the change of variables used above, so this problem can be cured by the simple expedient of differentiating twice in $\tau$, or applying the differential operator $D=\partial / \partial\left(t^{s}\right)=s^{-1} t^{1-s} \partial / \partial t$ twice if we stay with the original variable $t$. The resulting sum then has the value $\sum_{\nu \in \mathbb{Z}+\alpha} D^{2} f(\nu)$ with the same function $f(\nu)=f_{s, \beta}(\nu)$ as before, and since the function $D^{2} f(\nu)$, unlike $f(\nu)$ itself, is exponentially small both for large positive and for large negative values of $\nu$, we can now apply Poisson summation to equate this with a sum of Fourier transforms. But the differentiation and Fourier transformation operators are with respect to different variables, so the Fourier transform of $D^{2} f$ is simply $D^{2}$ of the Fourier transform $\widetilde{f}$ of $f$, which we have already calculated (for that calculation the parity of $s$ plays no role), so we get a formula for $D^{2}\left(L_{s}^{\text {sym }}\right)$ as a convergent sum of terms $D^{2} \widetilde{f}$, and then by integrating twice a formula for $L_{s}^{\text {sym }}$ itself as a sum of terms of exactly the same form as before. The only potential problem is that, since we have differentiated twice and then integrated twice, the final answer might be off by a linear function of $\tau$ (or of $t^{s}$ ). But from the results of $\S 3$ we already know the value of $L_{s}^{\mathrm{sym}}(\alpha, \beta ; t)$ up to terms that are smaller than any power of $t$, so we can read off the necessary constants of integration from the asymptotic formula given in the Proposition of $\S 3$, and since these contributions are already contained in the "elementary" part $E_{s}(\alpha, \beta ; t)$ in $(27)$, the "error" part is given by a formula exactly like the one for $s$ even, the only difference being that the symmetrization process is now slightly different from the one there. We do not write out the details, which are completely straightforward once one knows the trick of differentiating twice and then integrating twice. The final result corresponding to (31), but rewritten in a uniform way that applies to both even and odd $s$, takes the form

$$
\Lambda_{s}^{0}(\alpha, \beta ; \tau) \Lambda_{s}^{0}\left(-\alpha,(-1)^{s} \beta ; \tau\right)=\left(\text { elem. ) } \prod_{\substack{\mathfrak{z} \in \mathfrak{H} \\ \mathfrak{z}^{s} \tau= \pm 1}} \Lambda_{1 / s}^{0}(\mp \beta,-\alpha ; \mathfrak{z}) \Lambda_{1 / s}^{0}\left(\mp(-1)^{s} \beta, \alpha ; \mathfrak{z}\right) .\right.
$$


where "elem." denotes the elementary expression (which has the form of a constant times the product of exponentials in $\tau^{-1 / s}$ and $\tau$ ) obtained by exponentiating the negative of the right-hand side of (25) with $t^{s}=2 \pi \tau / i$.

In principle this finishes the calculation, but we still need to make one final modification to the definitions in order to put the formulas that we have obtained into the nicest form possible. When we looked above at the case of odd $s$, we essentially wrote the sum of the two convergent half-lattice sums defining $L_{s}^{\text {sym }}$ as the sum of a single divergent sum over the whole lattice and a divergent correction term defined formally as the sum over a half-lattice of a linear combination of 1 and $t^{s}$. But this latter expression has a standard "zeta regularization" by interpreting $\sum_{\nu \in(\mathbb{Z}+\alpha)_{>0}} \nu^{k}$ when $k \geq 0$ as $\bar{\zeta}(-k, \alpha)$. In fact, it is natural to make the corresponding renormalization even for the original convergent sum defining $L_{s}$, but now subtracting only one-half of the corresponding zeta regularization term, i.e., to replace the function $L_{s}(\alpha, \beta ; t)$ defined by (23) by the renormalized value

$$
L_{s}(\alpha, \beta ; t)-\pi i \bar{B}_{1}(\alpha) \bar{B}_{1}(\beta)-\frac{1}{2} \bar{\zeta}(-s, \alpha) t^{s}-\frac{\delta_{\alpha, 0}}{2} \log (1-\mathbf{e}(\beta)) .
$$

(Here we exclude the degenerate case $\alpha=\beta=0$ where one of the factors in the shifted product $\prod_{\nu}\left(1-\mathbf{e}(\beta) q^{\nu^{s}}\right)$ vanishes.) The symmetrization of this has a much simpler asymptotic form as $t \rightarrow 0$ than $L_{s}$ itself, since all but the first term and the term $\delta_{\beta, 0} s \log (1-\mathbf{e}(\alpha))$ in $(25)$ now disappear. In terms of the variable $\tau=i t^{s} / 2 \pi$ and the exponentiated form of $L_{s}$, the renormalization in question takes the form of replacing the function $\Lambda_{s}^{0}(\alpha, \beta ; t)$ defined in $(29)$ by

$$
\Lambda_{s}(\alpha, \beta ; \tau):=(1-\mathbf{e}(\beta))^{\delta_{a, 0} / 2} \mathbf{e}\left(\frac{\bar{B}_{1}(\alpha) \bar{B}_{1}(\beta)}{2}+\frac{\bar{\zeta}(-s, \alpha) \tau}{2}\right) \prod_{\substack{\nu \in \mathbb{Z}+\alpha \\ \nu>0}}\left(1-\mathbf{e}(\beta) q^{\nu^{s}}\right) .
$$

For the reason just explained, the symmetrization $\Lambda_{s}(\alpha, \beta ; \tau) \Lambda_{s}\left(-\alpha,(-1)^{s} \beta ; \tau\right)$ of $\Lambda_{s}$, which agrees with the function $\Theta_{s}(\alpha, \beta ; \tau)$ as defined by (31) if $s$ is even and could be taken as the "right" definition of $\Theta_{s}(\alpha, \beta ; \tau)$ also for $s$ odd, has a very simple asymptotic expression to all orders in $t$ as the product of a constant factor $(1-\mathbf{e}(\alpha))^{-\delta_{\beta, 0} s / 2}$ and a pure exponential $e^{c / t}$. But now if we observe that the definition (33) makes sense also for non-integral values of $s$, we can use it to also replace the factors $\Lambda_{1 / s}^{0}$ in the right-hand side of (32) by $\Lambda_{1 / s}$, and when we do this we find very pleasingly that the two remaining elementary factors are also absorbed by the renormalization. (For the verification of this, which we omit, one needs Hurwitz's functional equation relating $\operatorname{Li}_{1+1 / s}(\mathbf{e}(\beta))$ to $\bar{\zeta}(-1 / s, \beta)$.) In other words, if we replace every $\Lambda^{0}$ in (32) by the corresponding $\Lambda$, then the elementary "fudge factor" disappears entirely and we are left with the following much smoother statement, which is the main result of this paper.

Theorem 2. Let $s$ be a natural number and $(\alpha, \beta) \in(\mathbb{R} / \mathbb{Z})^{2} \backslash\{(0,0)\}$, and set $\left(\alpha^{*}, \beta^{*}\right)=$ $\left(-\alpha,(-1)^{s} \beta\right)$. Then for all $\tau \in \mathfrak{H}$ we have the identity

$$
\Lambda_{s}(\alpha, \beta ; \tau) \Lambda_{s}\left(\alpha^{*}, \beta^{*} ; \tau\right)=\prod_{\substack{\mathfrak{z} \in \mathfrak{H} \\ \mathfrak{z}^{s} \tau= \pm 1}} \Lambda_{1 / s}\left(\mp \beta, \alpha^{*} ; \mathfrak{z}\right) \Lambda_{1 / s}\left(\mp \beta^{*}, \alpha ; \mathfrak{z}\right) .
$$

The qualitative content of Theorem 2 is that the generalized theta-product $\Theta_{s}(\alpha, \beta ; \tau)$ can be expressed for every positive integer $s$ and all $\beta \neq 0$ as a rapidly convergent infinite sum of pure exponentials in $\tau^{-1 / s}$. As a corollary of this and of the decomposition (22) we obtain the corresponding assertion for the generalized eta-function $\eta_{s}(\tau)$, which was stated in a qualitative form in Theorem 1. The quantitative form expresses the correction factor $F_{s, \kappa}(t)$ occurring in Proposition 2 as a rapidly convergent infinite product. For the sake of simplicity we state it fully only for the most interesting case $s=2$ in the form of the following theorem giving an exact formula for $P_{2}(q)$ near an arbitrary root of unity, establishing and generalizing the formula found experimentally in $\S 2$ for its behaviour near a fifth root of unity. 
Theorem 3. Let $\kappa=a / c$ be a rational number with denominator $c$. Then

$$
P_{2}\left(\mathbf{e}(\kappa) e^{-1 / T^{2}}\right)=\frac{N_{2}(c) e^{C_{2}(\kappa) T}}{2 \pi T} \prod_{\substack{\ell(\bmod c), m>0 \\ m \equiv \pm a \ell^{2}(\bmod c)}} \frac{1}{1-\mathbf{e}(\ell / c) \exp \left(-\mathbf{e}(\mp 1 / 8)(2 \pi / c)^{3 / 2} \sqrt{m} T\right)}
$$

with $N_{2}(c)$ and $C_{2}(\kappa)$ defined as in Proposition 2.

The corresponding statement for $P_{s}\left(\mathbf{e}(\kappa) e^{-1 / T^{s}}\right)$ with $s>2$ is similar but more complicated, with extra terms $D_{s}(\kappa)$ and $\zeta(-s)$ as in Proposition 2, with $(2 \pi / c)^{3 / 2}$ replaced by $(2 \pi / c)^{1+1 / s}$, with the congruence on $n$ replaced by $n \equiv \pm a \ell^{s}(\bmod c)$, and with the 8 th roots of unity $\mathbf{e}(\mp 1 / 8)$ in the exponent replaced by appropriately chosen $(4 s)$-th roots of unity.

\section{Application to the sth power partition function}

We now return to the original reason for wanting to study the behaviour of the holomorphic function $\eta_{s}(\tau)$ near rational points, which is to try to obtain as exact a formula as possible for the number $p_{s}(n)$ of partitions of $n$ into $s$-th powers by the Hardy-Ramanujan circle method. In this final section we will describe some partial results in this direction, concentrating mostly on the case $s=2$ of partitions into squares, and contrasting the situation at each stage with the original one for $s=1$. We will derive an explicit formula describing the contributions to $p_{2}(n)$ (and in principle also $p_{s}(n)$ for $s>2$ ) coming via the circle method from neighbourhoods of all roots of unity, with each such contribution being a finite linear combination of Bessel-like functions. Whether this can eventually be refined to give an exact formula for $p_{s}(n)$, either as the nearest integer to some explicit finite sum as in the original paper of Hardy and Ramanujan, or even by a convergent infinite sum giving the value exactly as in the famous later paper [3] by Rademacher, still remains to be seen.

As already indicated, there are a number of differences to the original Hardy-Ramanujan situation, which for simplicity we will discuss in detail only for $p_{2}(n)$, the simplest case after ordinary partitions. Here the situation is simpler than the original one in a few respects (notably the absence of Dedekind sums and hence of the complicated arithmetic coefficients that Hardy and Ramanujan found), but are more subtle in several others. We describe them in turn, in numbered points of quite different lengths and degrees of technicality.

1. The first and in principle quite minor point, but nevertheless one that affects everything, is that $p_{2}(n)$ grows much more slowly than $p(n)$ (exponentially in $\sqrt[3]{n}$ rather than in $\sqrt{n}$ ), and $p_{s}(n)$ for $s>2$ yet more slowly (exponentially in $\sqrt[s+1]{n}$ ).

2. The second obvious but important point is that we now have electronic computers, whereas Hardy and Ramanujan had only the "practised and enthusiastic" computer Major McMahon (to quote the nice phrase by Hardy in his book on Ramanujan), so that while they had to be content with the values of $p(n)$ up to 200 , ending with the then very impressive number

$$
p(200)=3972999029388,
$$

we can find all of the values of $p_{2}(n)$ up to 100000 , ending with

$$
p_{2}(100000)=389977344281488282064568879923715009879905617086319698551970,
$$

in just over two seconds with the one-line GP/PARI program

$$
y=1+0(x)^{\wedge} 100001 ; \text { for }\left(n=1,316, y^{*}=1-x^{\wedge}\left(n^{\wedge} 2\right)\right) ; y=1 / y \text {; }
$$

and the definition $\mathrm{p} 2(\mathrm{n})=\operatorname{polcoef}(\mathrm{y}, \mathrm{n})$. 
3. The next point concerns the nature of the higher Bessel-like functions occurring in the expansion of $p_{s}(n)$. The idea of the circle method is to first write $p_{s}(n)$ by Cauchy's theorem as

$$
p_{s}(n)=\frac{1}{2 \pi i} \oint P_{s}(q) \frac{d q}{q^{n+1}}=\int_{\tau_{0}}^{\tau_{0}+1} P_{s}(\mathbf{e}(\tau)) \mathbf{e}(-n \tau) d \tau
$$

(integral over any path from $\tau_{0}$ to $\tau_{0}+1$ in $\mathfrak{H}$ ) and then break this integral up into a sum over short $\operatorname{arcs} A_{\kappa}$ near every rational point $\kappa$ modulo 1 ("major arcs") and a correction term coming from the rest of the circle ("minor $\operatorname{arcs}$ "). This decomposes $p_{s}(n)$ itself into a finite or infinite sum of contributions $p_{s, \kappa}(n)$ with $\kappa$ ranging over a finite subset or all of $\mathbb{Q} / \mathbb{Z}$ and a remainder term that has to be estimated. Proposition 2 expresses $P_{s}(q)$ at a point $q=\mathbf{e}(\kappa) e^{-T^{-s}}$ near a root of unity $\mathbf{e}(\kappa)$ as the sum of an elementary function of the form $c_{s}(\kappa) T^{-s / 2} \exp \left(C_{s}(\kappa) T+\frac{1}{2} \zeta(-s) T^{-s}\right)$ with a coefficient $c_{s}(\kappa)=\mathbf{e}\left(D_{s}(\kappa)\right)\left(N_{s}(\operatorname{den}(\kappa)) / 2 \pi\right)^{s / 2}$ depending only on $\kappa$ plus a much smaller (in fact, exponentially much smaller, as we saw in $\S 4)$ error term. This means that $p_{s, \kappa}(n)$ is the sum of a main term $p_{s, \kappa}^{(0)}(n)$ and a secondary term $p_{s, \kappa}^{(+)}(n)$ (discussed in 5. below), where $p_{s, \kappa}^{(0)}(n)$ is given as the integral over a suitable contour of $\frac{s c_{s}(\kappa)}{2 \pi i} T^{-1-3 s / 2} \exp \left(C_{s}(\kappa) T+\widetilde{n} T^{-s}\right)$ with $\widetilde{n}=n+\frac{1}{2} \zeta(-s)$ and hence has the form $c_{s}(\kappa) \widetilde{n}^{-3 / 2} H_{s}\left(C_{s}(\kappa) \widetilde{n}^{1 / s}\right)$ for some transcendental function $H_{s}(x)$. However, this function is not unique, because we have some freedom in the choice of the $\operatorname{arc} A_{\kappa}$ : to find the main contribution to the integral, we should let $A_{\kappa}$ pass through the "saddle" point in $\mathfrak{H}$ near $\kappa$ where $\left|q^{-n} P_{s}(q)\right|$ takes on its maximum (this point corresponds roughly to $\left.T=\left(s n / C_{s}(\kappa)\right)^{1 /(s+1)}\right)$ and then for purposes of optimal approximation should move from that point along the direction of steepest descent, but once we are away from the maximum we can deform the path in many ways, e.g. as a closed loop starting at $\kappa$ and passing through the saddle point, or into a path going from the saddle point to $-i \infty$ in the lower half-plane on both sides of $\kappa$. (We could not do this for the integral of $P_{s}(\mathbf{e}(\tau))$ itself, since it is not defined for $\tau$ in the lower half-plane, but we can for the elementary function approximating it.) The result of this freedom is that the function $H_{s}(x)$ itself is also not uniquely defined, though any two choices differ by an exponentially small quantity, so that the choice is not important in a Hardy-Ramanujan-like analysis where one takes a limited number of points $\kappa$ (they went up to denominator of the order of $n^{1 / 4}$ ) and has a far larger error than this anyway. It would, of course, be important if one wanted to get a Rademacher-like exact relation. In the classical case $s=1$ the functions $H_{1}(x)$ used by Hardy-Ramanujan and by Rademacher were, in our normalization,

$$
H_{1}^{\mathrm{HR}}(x)=\frac{1}{2 \sqrt{\pi}}\left(\sqrt{x}-\frac{1}{2}\right) e^{2 \sqrt{x}}, \quad H_{1}^{\mathrm{Rad}}(x)=\frac{1}{\sqrt{\pi}}\left(\sqrt{x}-\frac{1}{2}\right) \sinh (2 \sqrt{x}),
$$

respectively for $x$. For general $s$ different choices lead to any of the three functions

$$
\sum_{r=0}^{\infty} \frac{x^{r}}{r ! \Gamma\left(\frac{r}{s}-\frac{1}{2}\right)}, \quad s \sum_{r=0}^{\infty} \frac{x^{r s}}{(r s) ! \Gamma\left(r-\frac{1}{2}\right)}, \quad s \sum_{r=0}^{\infty} \frac{x^{r s+3 s / 2}}{r ! \Gamma\left(r s+\frac{3 s}{2}+1\right)},
$$

of which the second is obtained from the first by taking $s$ times the sum of the terms with $r$ divisible by $s$ and the third from the second by shifting $r$ by $\frac{3 s}{2}$. (We do not give the details of the calculations, since as already remarked the specific choice of $H_{s}$ will not play any role within the accuracy of the calculations we are describing.) These three functions are all different in general, but it is amusing to notice that in the classical case $s=1$ the first two coincide but that the two remaining functions $\frac{1}{\sqrt{\pi}}\left(\sqrt{x} \sinh (2 \sqrt{x})-\frac{1}{2} \cosh (2 \sqrt{x})\right)$ and $\frac{1}{\sqrt{\pi}}\left(\sqrt{x} \cosh (2 \sqrt{x})-\frac{1}{2} \sinh (2 \sqrt{x})\right)$ are different from both $H_{1}^{\mathrm{HR}}(x)$ and $H_{1}^{\mathrm{Rad}}(x)$. In our main case $s=2$, we will choose the third of the functions listed above, but only for $\Re(x)>0$, i.e., we will define $H_{2}(x)$ by

$$
H_{2}(x)=2 \sum_{r=0}^{\infty} \frac{x^{2 r+3}}{r !(2 r+3) !} \quad \text { if } \Re(x)>0, \quad H_{2}(x)=0 \quad \text { if } \Re(x)<0,
$$


where the case distinction arises because the contour integral that originally defines $H_{2}(x)$ can be moved to infinity without crossing the singularity at $T=0$ if $\Re(x)<0$ but not if $\Re(x)>0$. The main contribution $p_{2, \kappa}^{(0)}(n)$ to $p_{2, \kappa}(n)$ is then given by

$$
p_{2, \kappa}^{(0)}(n)=\frac{N_{2}(\operatorname{den}(\kappa))}{2 \pi n^{3 / 2}} \mathbf{e}(-n \kappa) H_{2}\left(C_{2}(\kappa) \sqrt{n}\right) .
$$

To deduce from this the precise asymptotic behaviour of $p_{2, \kappa}^{(0)}(n)$, we use the asymptotic formula

$$
H_{2}\left(2 X^{3 / 2}\right)=\frac{X e^{3 X}}{\sqrt{12 \pi}}\left(1-\frac{17}{36 X}-\frac{35}{2592 X^{2}}-\cdots\right)
$$

for $X$ large, which is easily proved using an integral representation or ODE of the function $\mathrm{H}_{2}$.

4. The next point is much more interesting. In the case $s=1$ studied by Hardy and Ramanujan, the number $C_{s}(\kappa)=C_{1}(\kappa)$ occurring in the exponent in Proposition 2 has the very simple form $\zeta(2) / c^{2}$, where $c$ as usual denotes the denominator of $\kappa$. This means that the contributions coming from different rational numbers ("cusps") with the same denominator are all of the same order of magnitude and that this size decreases monotonically and rapidly as $c$ grew, leading for $n=200$ to the famous table of Hardy-Ramanujan that began

$$
\begin{array}{r}
3972998993185.896 \\
36282.978 \\
-87.555 \\
5.147 \\
1.424
\end{array}
$$

and whose first five terms already gave $p_{2}(200)$ as the nearest integer. But in our case that does not happen at all, since the coefficients $C_{2}(\kappa)$ depend erratically on $\kappa$ and can even have real parts of different signs for $\kappa$ with the same denominator, as we already saw in $\S 2$ in the case of denominator 5 . Thus when we look, for instance, at the the sum of the contributions from $\kappa$ with denominator $c=1, \ldots, 5$ to the number $p_{2}\left(10^{5}\right)$ given above, they have the approximate values $3.9 \cdot 10^{59}, 1.8 \cdot 10^{26}$, $-2.0 \cdot 10^{3},-8.3 \cdot 10^{32}$, and $2.9 \cdot 10^{15}$, respectively, of wildly varying sizes. In fact the biggest contributions apart from the one from $\kappa=0$ come from $\kappa=1 / 4$ and $\kappa=3 / 4$ (i.e., from $q$ near $i$ or $-i$ ), so that the difference $\Delta_{2}(n)$ between $p_{2}(n)$ and the leading term $p_{2,0}^{(0)}(n)$ in the circle method approximation to it has an approximate size that depends on $n(\bmod 4)$, or more precisely, that changes sign when $n$ increases by 2 , as one can see clearly in the following table

$$
\begin{array}{llll}
-8.3104 \cdot 10^{32}, & -1.3710 \cdot 10^{32}, & 8.3151 \cdot 10^{32}, & 1.3730 \cdot 10^{32}, \\
-8.3198 \cdot 10^{32}, & -1.3750 \cdot 10^{32}, & 8.3244 \cdot 10^{32}, & 1.3771 \cdot 10^{32}, \\
-8.3291 \cdot 10^{32}, & -1.3791 \cdot 10^{32}, & 8.3338 \cdot 10^{32}, & 1.3811 \cdot 10^{32}, \\
-8.3384 \cdot 10^{32}, & -1.3831 \cdot 10^{32}, & 8.3431 \cdot 10^{32}, & 1.3852 \cdot 10^{32}
\end{array}
$$

giving the values of 16 consecutive values $\Delta_{2}\left(10^{5}\right), \ldots, \Delta_{2}\left(10^{5}+15\right)$ grouped in groups of four to make this near-periodicity more visible. (We also see from this table that the term $p_{2,0}^{(0)}(n)$ of the approximation coming from the principal cusp $k=0$ already gives $p_{2}(N)$ correctly in this range to about 27 significant digits, whereas the exponential of the Hardy-Ramanujan asymptotic formula for $\log \left(p_{2}(N)\right)$ that we quoted in (3) would give a value that is more than twelve million times too big.) To see why this is true, we give a table of values of the first numbers $C_{2}(\kappa)$, ordered by their real 
parts. This table begins

\begin{tabular}{c|ccccc}
$\kappa$ & 0 & $\pm \frac{1}{4}$ & $\frac{1}{2}$ & $\pm \frac{1,4,7}{9}$ & $\pm \frac{1}{8}$ \\
\hline$C_{2}(\kappa)$ & 2.315 & $1.038 \pm 0.383 i$ & 0.819 & $0.709 \pm 0.069 i$ & $0.611 \pm 0.477 i$
\end{tabular}

\begin{tabular}{c|ccccc}
$\kappa$ & $\pm \frac{1,9}{16}$ & $\pm \frac{1,4,6,9,11}{25}$ & $\pm \frac{1}{3}$ & $\pm \frac{1}{5}$ & $\pm \frac{1}{12}$ \\
\hline$C_{2}(\kappa)$ & $0.590 \pm 0.360 i$ & 0.461 & $0.446 \pm 0.360 i$ & 0.440 & $0.427 \pm 0.363 i$
\end{tabular}

\begin{tabular}{c|ccccc}
$\kappa$ & $\pm \frac{2,3,7,8,12}{25}$ & $\pm \frac{5,13}{16}$ & $\pm \frac{1}{24}$ & $\pm \frac{1,13,25}{36}$ & $\pm \frac{1,9,17,25}{32}$ \\
\hline$C_{2}(\kappa)$ & 0.419 & $0.418 \pm 0.119 i$ & $0.378 \pm 0.398 i$ & $0.362 \pm 0.222 i$ & $0.331 \pm 0.298 i$
\end{tabular}

(we have listed all values with $\left.\Re\left(C_{2}(\kappa)\right)>0.32\right)$ and clearly explains why the various cusps $\kappa$ have contributions to $p_{2}(n)$ of such unpredictably varying sizes. For $n=10^{5}$, the 17 denominators whose total primary contribution $\sum_{\operatorname{den}(\kappa)=c} p_{2, \kappa}^{(0)}(n)$ to $p_{2}(n)$ are largest in absolute value, sorted by that absolute value, are $c=1,4,2,8,16,3,25,12,5,24,32,48,40,64,20,96$, and 17 . (The individual contributions from $c=9$ are larger in absolute value than those from $c=8$, but they sum to zero.) The sum of these contributions differs from $p_{2}\left(10^{5}\right) \approx 3.90 \cdot 10^{59}$ by only $8.26 \cdot 10^{13}$, so these 17 values of $c$ already give an excellent approximation. But if we continue to the next smaller terms, coming from $c=80,56,120,28,100,60,13, \ldots$, then they are all much smaller than the number $8.26 \cdot 10^{13}$, which remains constant to this accuracy no matter how far we go with $c$. This remaining discrepancy comes from the secondary terms already mentioned in $\mathbf{3 .}$, to which we now turn.

5. The last point is the most subtle one. Because $\eta(\tau)$ is a modular function, the generating function $1 / \eta(\tau)$ of partitions has an expansion near a rational number $\kappa=a / c$ given up to an innocuous factor (a constant times $\sqrt{\tau-\kappa}$ ) by $\eta(\gamma(\tau))^{-1}=Q^{-1 / 24}+Q^{23 / 24}+\cdots$ with $Q=e^{2 \pi i \gamma(\tau)}$ (where $\left.\gamma(\infty)=\kappa\right)$, and this always has precisely one exponentially large term $Q^{-1 / 24}$ as $\tau$ tends to $\kappa$. This means that in both the Hardy-Ramanujan approximation and the Rademacher exact formula only the leading term (arithmetic function times exponential) coming from each $\kappa$ plays any role. But for higher $s$ this is no longer true. In particular, Theorem 3 can be written in the form

$$
P_{2}\left(\mathbf{e}(\kappa) e^{-1 / T^{2}}\right)=\frac{N_{2}(c)}{2 \pi T} \sum_{\mu: X_{\kappa} \rightarrow \mathbb{Z}_{\geq 0}} \mathbf{e}(L(\kappa, \mu) / c) e^{C_{2}(\kappa, \mu) T}
$$

where $X_{\kappa}$ is defined by

$$
X_{a / c}=\left\{(\ell, m) \in(\mathbb{Z} / c \mathbb{Z}) \times(\mathbb{Z} \backslash\{0\}) \mid \ell^{2} a \equiv m(\bmod c)\right\}
$$

and the sum is over all functions from it to $Z_{\geq 0}$ (these are the exponents of the various terms when we expand the product in Theorem 3 as a product of geometric series), with $L(\mu, \kappa) \in \mathbb{Z} / c \mathbb{Z}$ and $C_{2}(\kappa, \mu) \in \mathbb{C}$ defined by

$$
L(\kappa, \mu)=\sum_{(\ell, m) \in X_{\kappa}} \mu(\ell, m) \ell, \quad C_{2}(\kappa, \mu)=C_{2}(\kappa)-\left(\frac{2 \pi}{c}\right)^{3 / 2} \sum_{(\ell, m) \in X_{\kappa}} \mu(\ell, m) \frac{|m|-i m}{\sqrt{2|m|}} .
$$

Since there are only finitely many pairs $(\ell, m)$ with $(2 \pi / c)^{3 / 2} \sqrt{|m| / 2}<\Re\left(C_{2}(\kappa)\right)$, this gives us a finite expression

$$
p_{2, \kappa}(n)=\frac{N_{2}(c)}{2 \pi n^{3 / 2}} \mathbf{e}(-n \kappa) \sum_{\substack{\mu: X_{\kappa} \rightarrow \mathbb{Z}^{+} \\ \Re\left(C_{2}(\kappa, \mu)\right)>0}} \mathbf{e}(L(\kappa, \mu) / c) H_{2}\left(C_{2}(\kappa, \mu) \sqrt{n}\right)
$$


(here we could even omit the inequality since we have defined $H_{2}(x)$ to be 0 if $\Re(x)<0$ ) for the contribution from the cusp $\kappa$ to $p_{2}(n)$. As already mentioned in 3., we can now split this into a main term $p_{2, \kappa}^{(0)}(n)$ corresponding to $\mu=0$ and a secondary term $p_{2, \kappa}^{(+)}(n)$ corresponding to all other values of $\mu$. But unlike the situation for $s=1$, this secondary term no longer vanishes identically. As a numerical example, we take $n=10^{5}$ and consider the numbers $\kappa= \pm \frac{1}{9}, \pm \frac{4}{9}$ and $\pm \frac{7}{9}$ of denominator 9 , where we have grouped them in pairs differing by sign in order to have only real contributions. The corresponding primary contributions to $p_{2}(n) \approx 3.9 \cdot 10^{59}$ have values approximately equal to $-3.39 \cdot 10^{23},-1.15 \cdot 10^{23}$ and $4.53 \cdot 10^{23}$, respectively, but these actually sum to zero (this is connected with the fact that 9 is a perfect square), so that the total primary contribution to $p_{2}\left(10^{5}\right)$ coming from $\kappa$ with denominator 9 vanishes. But there are secondary contributions coming from $\mu$ supported at $(\ell, m)$ with $m=1$ or $m=-2$ in each case, and the total secondary contribution from the denominator $c=9$ to the value of $p_{2}\left(10^{5}\right)$ then turns out to be the non-zero number $8.30 \cdot 10^{13}$, very close to the amount $8.26 \cdot 10^{23}$ that was still missing at the end of point 4 . above. In fact, $c=9$ turns out to give by far the largest secondary contribution in this case. In particular, for $\kappa$ with denominators up to 7 there are no non-zero multiplicity functions $\mu$ at all with $\Re\left(C_{2}(\kappa, \mu)\right)$ positive. For $\kappa= \pm 1 / 8$ there are four such $\mu$ (for $\kappa= \pm 3 / 8$ there are none), but they are given by $\mu$ supported at $(\ell, \mp 1)$ with $\ell=1$, 3,5 and 7 modulo 8 , and the sum of the corresponding coefficients $\mathbf{e}(L(\kappa, \mu) / 8)=\mathbf{e}(\ell / 8)$ therefore vanishes because the sum of the primitive 8th roots of unity is 0 . If we continue to larger values of $c$, then we find contributions of the order of $10^{11}$ from $c=25$ and $c=36$, with the remaining ones yet smaller. Adding up all of the secondary contributions as far as I calculated gave a total of about $8.25 \times 10^{13}$, accounting for very nearly all of the missing amount, and I assume that the remaining very small discrepancy is a consequence of the numerical analysis and not something intrinsic.

Note added in proof. After an earlier version of this paper was finished and accepted for publication, I discovered results in [2] that I had overlooked on a first reading. In the introduction to the paper, the authors discuss the problem of partitions into sums of squares of higher powers, and the asymptotic formula (3) for the logarithm of $p_{s}(n)$ is displayed prominently. But at the very end of of the paper (p. 111 in subsection 7.3) they return to this problem as an example of a "wide range of problems to which our results are partly applicable," and there they give an asymptotic formula for $p_{s}(n)$ as opposed to merely its logarithm. This formula gives only the leading term, coming from the behaviour of $P_{s}(q)$ near $q=1$ and from the main exponential approximating the function $H_{s}(n)$, so that, for instance, in the case $s=2, n=10^{5}$ used as an example in $\S 5$ of this paper, their formula would give the correct answer only within about one percent as opposed to the 27-digit accuracy achieved by computing the whole contribution from $\kappa=1$ or the 48-digit accuracy achieved by including contributions from all cusps. But of course it is still vastly more precise than the equation (3) that I quoted, which as mentioned would be off by a factor of more than $10^{7}$ in this example. More importantly, they explicitly gave equation (5) relating $\eta_{2}(-1 / \tau)$ to a product of two values of $\eta_{1 / 2}$, though it is expressed rather differently, so that this result, which was the discovery that gave rise to this whole paper, had actually been known a century earlier. The more general equation (6) for $s>2$ does not seem to be given explicitly in their paper, although it is clear that they could have obtained it, and more importantly there is nothing about the behaviour at other cusps than $\kappa=0$, so nothing like our Theorems 1 and 3, and similarly no discussion of the analogue for higher power partitions of the full formula for $p_{s}(n)$ as a sum of contributions from all cusps. I therefore hope that the results of this paper are still of some interest. In the end I chose not to rewrite my text since the description of the numerical approach that I used to "discover" (but actually to rediscover) equation (5) sets the stage for the much more complicated analysis needed in the general case.

Acknowledgement. I would like to thank Fabio Novaes, whose question whether I could say anything about the analytic properties of the function $P_{s}(q)$, which had turned up in his work on the Chern-Simons formulation of 3D gravity and its connection to integrable systems, was the 
original motivation for the present investigation. I would also like to thank Sameer Murthy and Stavros Garoufalidis for many very helpful discussions. A joint paper with them on a related topic is currently in preparation.

\section{References}

[1] S. Garoufalidis and D. Zagier, Knots and their related q-series, Preprint (2021)

[2] G.H. Hardy and S. Ramanujan, Asymptotic formula in combinatory analysis, Proc. London Math. Soc., (2) 17, (1918), 75-115. Reprinted in Collected Papers of Srinivasa Ramanujan, Chelsea Publishing Company, New York (1962), pp. 276-309 and in Collected Papers of G.H. Hardy; Including Joint Papers with J.E. Littlewood and Others, Vol. I, Clarendon Press, Oxford (1972), 306-339.

[3] H. Rademacher, On the partition function p(n), Proc. Lond. Math. Soc. 43 (1937), 241-254.

[4] D. Zagier, The Mellin transform and other useful analytic techniques, Appendix to E. Zeidler, Quantum Field Theory I: Basics in Mathematics and Physics. A Bridge Between Mathematicians and Physicists, Springer-Verlag, Berlin-HeidelbergNew York (2006), 305-323.

\section{Don Zagier}

Max Planck Institute for Mathematics

Bonn, Germany

and

International Centre for Theoretical Physics

Trieste, Italy

e-mail:dbz@mpim-bonn.mpg.de 